\title{
Evaluation of reactivation of HSVI, HHV6, CMV and EBV in a population of patients undergoing allogeneic bone marrow transplantation
}

\author{
Caterina Matinato, Giovanna Lunghi, Anna Orlandi, Silvia Melotti, Antonella Zoccoli, \\ Damiano Picicco, Erminio Torresani \\ UOS Virologia, Laboratorio centrale di analisi, Fondazione IRCCS Ca' Granda Ospedale Maggiore Policlinico di Milano
}

Key words: Bone marrow transplantation, Herpes virus reactivation, aGVDH

\begin{abstract}
Valutazione della riattivazione di HSVI, HHV6, CMV ed EBV in una popolazione di pazienti sottoposti a trapianto allogenico di midollo
\end{abstract}

\section{SUMMARY}

Herpes viruses are recognized as important pathogens as a result of viral reactivation in immunocompromised hosts, especially in patients undergoing bone marrow transplantation. Objectives of this study were the assessment of the reactivation of herpes virus HSVI, HHV6, CMV and EBV and the correlation between viral reactivation and progression of transplantation in a population of patients undergoing allogeneic bone marrow transplantation at Fondazione IRCCS Ospedale Maggiore Ca' Granda Policlinico, Milan.

Viral DNA was detected and quantified by Real Time PCR in a population of 35 patients undergoing allogeneic bone marrow transplantation. The viral reactivation was observed in 7 patients for HSVI (20\%), 6 patients for HHV6 (17.1\%), II patients for CMV (3I.4\%) and 4 patients for EBV (II.4\%). Difference in the incidence of aGVDH between patients with viral reactivation versus those for which there was no reactivation was statistically significant. These data confirm the importance of monitoring viral load for the management of antiviral therapy in order to prevent CMV disease and complications related to herpes viruses reactivation.

\section{INTRODUZIONE}

La riattivazione dell'infezione latente da virus erpetici, costituisce un rischio molto elevato per i pazienti sottoposti a trapianto allogenico di midollo, a causa delle condizioni di granulocitopenia, abbattimento delle difese immunitarie sia di tipo cellulo-mediato che umorale e aumentata incidenza di aGVHD (Graft Versus Host Disease acuta). La riattivazione dei diversi tipi virali è associata con il periodo temporale connesso al trapianto che è suddiviso in 4 fasi correlate con un diverso stadio di immunosoppressione $(9,10)$ :

- Pretrapianto

- Preengraftment (approssimativamente da 0 a 30 giorni dopo il trapianto)

- Postengraftment (approssimativamente da $30 \mathrm{a}$ 100 giorni dopo il trapianto)

- Periodo tardivo del post trapianto ( $\geq 100$ giorni dopo il trapianto)

Preengraftment: è definito come il momento in cui il numero assoluto dei granulociti è $<500 / \mu 1 \mathrm{e}$ il numero di piastrine è $<20.000 \times 10^{6}(1,8)$. Durante questo periodo la riattivazione più frequente è quella da HSV (Herpes Simplex Virus), seguita dalla riattivazione del virus HHV6 (Human Herpes Virus 6) (6).
Postengraftment: generalmente in questa fase si avvia la risoluzione della neutropenia grave che dovrebbe stabilizzarsi entro i 100 giorni dal trapianto. Sul piano delle difese immunitarie, il deficit dell'immunità cellulo-mediata è particolarmente rilevante, pertanto la riattivazione dei virus erpetici ed in particolare del Citomegalovirus (CMV), rappresenta l'evento infettivo più importante evidenziato in questo periodo.

Periodo tardivo del post trapianto: è definito come il periodo durante il quale si assiste al recupero della funzionalità del sistema immunitario, sia sul versante dell'immunità cellulo-mediata che umorale. In questa fase si osservano frequentemente manifestazioni cliniche legate alla slatentizzazione di VZV (Varicella Zooster Virus) ed EBV (Virus di Epstein-Barr).

Anche la presenza di DNA di CMV è frequentemente osservabile ed è correlabile all'entità della ricostituzione immunitaria.

La prevenzione delle infezioni da virus erpetici si basa sulla somministrazione di una terapia antivirale a scopo profilattico e sull'approntamento di una terapia preemptive per la prevenzione della riattivazione di CMV. A questo proposito il monitoraggio della carica virale è di importanza fonda-

\section{Corresponding author: Caterina Matinato}

UOS Virologia, Fondazione IRCCS Cà Granda Ospedale Maggiore Policlinico - Milano

Via F. Sforza, 35 - 20122 Milano

Tel.: 02 550334I5; Fax: 02 5503337। 
mentale nella gestione del paziente.

Obiettivi del nostro lavoro sono stati la valutazione dell'incidenza di riattivazione dei virus erpetici HSV1, HHV6, CMV ed EBV e la correlazione tra riattivazione virale e decorso del trapianto (aGVHD e sopravvivenza) in una popolazione di pazienti sottoposti a trapianto allogenico di midollo presso le strutture della Fondazione IRCCS $\mathrm{Ca}$ ' Granda Ospedale Maggiore Policlinico di Milano. Il virus VZV, non è stato oggetto di questo studio in quanto il protocollo relativo alla tempistica di ricerca del DNA virale non ha seguito le stesse modalità descritte per gli altri virus erpetici. L'approvazione da parte del comitato etico non si è resa necessaria per il presente lavoro, in quanto sono stati utilizzati dati raccolti a seguito di esami eseguiti di routine

\section{MATERIALI E METODI Popolazione}

Per lo studio di natura prospettica, è stata utilizzata una popolazione costituita da 35 pazienti sottoposti a trapianto allogenico di midollo nel periodo 2007-2010. Per 21 pazienti il midollo proveniva da fratello HLA (Human Leukocyte Antigen) compatibile (SIB), per 14 da donatore non consanguineo HLA compatibile (MUD) a questi ultimi veniva somministrato siero antilinfocitario. L'età media dei pazienti era di 43 anni (range: 18-65) 19 maschi e 16 femmine, la diagnosi di ammissione dei pazienti è elencata nella Tabella 1 .

A tutti i pazienti veniva somministrato aciclovir per la profilassi anti HSV a partire da 5 giorni fino a 6-9 mesi dopo il trapianto, mentre a tutti i pazienti per i quali era evidenziata una carica virale di CMV >10.000 genomi/ml veniva somministrata una terapia preemptive a base di ganciclovir o valganciclovir.

\section{Ricerca DNA virale}

È stata eseguita su tutti i pazienti una volta alla settimana per le prime quattro settimane, in seguito una volta al mese per 6-9 mesi. Il DNA di CMV ed EBV è stato estratto a partire da sangue intero $(100 \mu l)$ mentre il DNA di HHV6 ed HSV1 è stato estratto da plasma $(500 \mu \mathrm{l})$. L'estrazione del DNA è stata effettuata mediante sistema automatizzato NucliSENS EasyMAG bioMérieux basato sull'utilizzo di silice magnetica la cui funzione è quella di trattenere gli acidi nucleici: i campioni vengono miscelati con un tampone di lisi contenente un agente caotropico che lisa le cellule e le particelle virali liberando il DNA virale che viene catturato dalla silice magnetica, purificato, rilasciato e concentrato in $55 \mu \mathrm{l}$ di tampone di eluizione. Per l'amplificazione e la quantificazione del DNA virale è stato utilizzato il kit REAL-TIME Alert Q-PCR NANOGEN che prevedeva l'amplifica- zione delle seguenti regioni specifiche per ogni virus:

- CMV: regione codificante per il Major Immediate Early Antigen (MIEA)

- HSV1: regione codificante per la Glicoproteina D

- HHV6: regione ORF 13

- EBV: regione codificante per l'antigene nucleare (EBNA1).

Per tutte le reazioni di amplificazione, il kit utilizzava un controllo interno (regione codificante per il gene della beta globina umana) amplificato contemporaneamente al target.

I risultati sono stati espressi come genomi $/ \mathrm{ml}$. L'analisi statistica dei dati è stata effettuata mediante test esatto di Fisher.

\section{RISULTATI}

Su 35 pazienti sottoposti a trapianto allogenico di midollo la riattivazione virale è stata evidenziata in 7 pazienti per HSV1 $(20 \%), 6$ pazienti per HHV6 (17.1\%), 11 pazienti per CMV (31.4\%) e 4 pazienti per EBV (11.4\%). La presenza di DNA di HHV6 è stata evidenziata in un solo campione per ognuno dei 6 pazienti, mentre per quanto riguarda HSV1 su 3 pazienti si sono evidenziati più campioni positivi, 10 pazienti avevano ripetute positività per CMV e 3 pazienti avevano ripetute positività per EBV. I valori medi di carica virale rilevati erano rispettivamente: HSV1: 6885 geno$\mathrm{mi} / \mathrm{ml}(\mathrm{min}<500$ - $\max$ 53424), HHV6: 4914 genomi/ml (min $600-\max 20166), \mathrm{CMV}: 6770$ genomi $/ \mathrm{ml}(\min <500-\max 62535)$ EBV: 197272 genomi/ml $(\min <500-\max 1987970)$, tutti valori abbastanza contenuti se si fa eccezione per il numero di genomi/ml di EBV il cui valore medio peraltro risente dell'elevato numero di genomi $/ \mathrm{ml}$ rilevati in due campioni provenienti dallo stesso paziente.

Gli episodi di coinfezione tra i diversi herpes virus sono stati rilevati su uno scarso numero di campioni, infatti in 2 campioni su $35(5.7 \%)$ è stata rilevata una coinfezione tra $\mathrm{CMV} / \mathrm{HSV}$, la stessa percentuale è stata rilevata per la coinfezione tra CMV/HHV6 e CMV/EBV, mentre in 1 campione $(2.9 \%)$ è stata rilevata una coinfezione tra HHV6 ed EBV.

Le percentuali di riattivazione virale in dipendenza del periodo post trapianto sono evidenziate nella Tabella 2, mentre i dati relativi all'incidenza della aGVHD ed alla sopravvivenza dei pazienti, in funzione della riattivazione virale sono indicati nella Tabella 3. Il test esatto di Fisher ha evidenziato una differenza significativa $(\mathrm{p}=0.035)$ tra l'incidenza della aGVDH nei pazienti con riattivazione virale rispetto a quelli per i quali non si è avuta riattivazione, mentre la presenza di DNA virale non influenza la sopravvivenza $(\mathrm{p}=0.3)$. Per 
quanto riguarda il contributo dei diversi tipi virali all'incidenza della aGVDH ed alla sopravvivenza dei pazienti, il DNA di HSV1, HHV6, CMV ed EBV è stato rilevato rispettivamente nel 5.7\%, $11.4 \%, 20 \%$ ed $8.6 \%$ dei pazienti con aGVDH, mentre HSV1 ed HHV6 sono stati identificati nell' $8.6 \%$, CMV nel $25.7 \%$ ed EBV nel $11.4 \%$ dei pazienti deceduti. Il diverso tipo di donatore di midollo, SIB o MUD, non incide sui dati di sopravvivenza, aGVHD e riattivazione virale, in quanto non sono state rilevate differenze significative tra $\mathrm{i}$ due gruppi di pazienti (dati non mostrati).

Tabella I.

\begin{tabular}{ll}
\hline Diagnosi di ammissione & N. Pazienti \\
\hline Linfoma di Hodgkin & 2 \\
\hline Leucemia linfoblastica acuta & 7 \\
\hline Leucemia linfatica cronica & $\mathrm{I}$ \\
\hline Leucemia mieloide acuta & 7 \\
\hline Leucemia mieloide acuta e linfatica cronica & $\mathrm{I}$ \\
\hline Leucemia mielomonocitica acuta evoluta in leucemia mieloide acuta & $\mathrm{I}$ \\
\hline Linfoma non-Hodgkin & 8 \\
\hline Linfoma non-Hodgkin a cellule T/ Micosi fungoide & $\mathrm{I}$ \\
\hline Mielofibrosi & 3 \\
\hline Mielofibrosi idiopatica e leucemia linfatica cronica & $\mathrm{I}$ \\
\hline Mielofibrosi idiopatica e leucemia mieloide acuta & $\mathrm{I}$ \\
\hline Mieloma multiplo & 2 \\
\hline
\end{tabular}

Tabella 2. Percentuali di riattivazione virale

\begin{tabular}{lccc}
\hline & Preengraftment & Postengraftment & Periodo tardivo post trapianto \\
\hline HSVI & $50 \%$ & $25 \%$ & $25 \%$ \\
\hline HHV6 & $66.7 \%$ & & $33.3 \%$ \\
\hline CMV & $16.1 \%$ & $18.1 \%$ & $65.8 \%$ \\
\hline EBV & & $16.7 \%$ & $83.3 \%$ \\
\hline
\end{tabular}

Tabella 3. Sopravvivenza

\begin{tabular}{|c|c|c|c|c|c|c|c|}
\hline \multirow{2}{*}{ DNA virale } & \multicolumn{4}{|c|}{ aGVDH } & \multicolumn{3}{|c|}{ Sopravvivenza } \\
\hline & & Sì & & Vo & & eduti & Viventi \\
\hline Presente & 10 & $(76.9 \%)$ & 8 & $(36.4 \%)$ & II & $(61.1 \%)$ & $7 \quad(4 I .2) \%$ \\
\hline Assente & 3 & $(23.1 \%)$ & 14 & $(63.6 \%)$ & 7 & (38.9\%) & $10(58.8 \%)$ \\
\hline Totale & & 13 & & 22 & & 18 & 17 \\
\hline
\end{tabular}

\section{DISCUSSIONE E CONCLUSIONI}

Nonostante i molti progressi sia in ambito diagnostico che terapeutico nella gestione delle infezioni da virus erpetici nei soggetti sottoposti a trapianto di midollo, la morbilità e la mortalità risultano essere ancora piuttosto elevate, soprattutto se si considerano le problematiche legate alla riattivazione del CMV che è il virus più frequentemente rilevato in questo gruppo di pazienti (5).

È stato osservato come la riattivazione da $\mathrm{CMV}$, comporti la presenza in circolo del DNA virale anche per lunghi periodi in assenza di una terapia mirata, mentre di contro, la riattivazione del HHV6 è in genere correlata con la presenza di un solo risultato positivo (2).

La riattivazione virale nella popolazione oggetto di questo studio, mostra dati simili a quanto su riportato, infatti, 10/11 (90.9\%) pazienti positivi per CMV DNA, hanno avuto più di un campione positivo, mentre in tutti i pazienti positivi per HHV6 il DNA è stato rilevato in un solo campione. Anche la presenza del DNA di HSV1 ed EBV è stata rilevata in alcuni pazienti su più campioni. Le cariche virali erano piuttosto contenute, essendo i valori medi inferiori ai 10.000 genomi $/ \mathrm{ml}$, se si fa eccezione per un paziente che ha avuto su due campioni un numero elevato di genomi $/ \mathrm{ml}$ di EBV DNA.

Per quanto riguarda la tempistica di riattivazione, HSV1 ed HHV6 si ritrovano prevalentemente nelle prime settimane dopo il trapianto durante la fase preengraftment, mentre CMV pur essendo rilevato in tutte e tre le fasi del post trapianto, è più frequentemente identificato nel periodo tardivo, probabilmente a causa della non ancora effettiva ricostituzione della risposta immunitaria.

La riattivazione del virus EBV è stata identificata in pochi pazienti, quasi tutti nel periodo tardivo del post trapianto. In generale, le percentuali di riattivazione virale sono piuttosto contenute, soprattutto se si fa riferimento alla riattivazione da HHV6 che è stimata essere tra il $28 \%$ ed il $75 \%$ nei soggetti sottoposti a trapianto di midollo (4), mentre nella nostra popolazione la riattivazione è stata osservata nel $17.1 \%$ dei campioni.

Non sembra esserci sinergia tra $i$ diversi virus erpetici, in quanto la coinfezione tra i diversi tipi virali è stata osservata in una scarsa percentuale di campioni, probabilmente l'efficacia della terapia antivirale preventiva e della terapia preemptive nei confronti del CMV possono spiegare sia il numero contenuto di campioni positivi che le basse cariche virali ritrovate $(7,11)$.

La presenza di DNA virale, correla positivamente 
con la presenza di aGVDH ed in particolare, questa correlazione è dovuta in massima parte alla presenza del CMV, infatti il $20 \%$ dei pazienti con presenza di CMV sperimenta una aGVDH di grado $\geq$ I (3) di contro, la sopravvivenza dei pazienti non è influenzata dalla positività al DNA virale anche se CMV è comunque il virus rilevato più frequentemente nei pazienti deceduti.

In conclusione, i dati rilevati confermano l'importanza sia della terapia preventiva che del monitoraggio della carica virale ai fini dell'impostazione della terapia preemptive per la profilassi della malattia da CMV inoltre, data l'importanza che la riattivazione virale ha nell'incidenza della aGVDH, il monitoraggio del DNA virale fornisce informazioni fondamentali nella gestione di questa complicanza.

\section{BIBLIOGRAFIA}

1. CDC. Guidelines for preventing opportunistic infections among hematopoietic stem cell transplant recipients. MMWR Recomm Rep. Oct 2000; 49 (RR-10): 1125, CE1-7.

2. Chan PKS, Peiris JSM, Yuen KY, et al. Human herpes virus 6 and human herpes virus 7 infections in bone marrow transplant recipients. Journal of medical virology, 1997; 53: 295-305.

3. Choi SW, Levine JE, Ferrara JLM. Pathogenesis and management of graft-versus-host disease. Immunol Allergy Clin N Am 2010; 30: 75-101.

4. De Bolle L, Naesens N, De Clerq E. Update on Human Herpes Virus 6 biology, clinical features, and therapy. Clinical Microbiology Reviews jan 2005: 217-245.

5. Jaskula E, Dlubek D, Sedzimirska $M$, et al. Reactivations of Cytomegalovirus, Human Herpes Virus 6 and Epstein Barr Virus differ with respect to risk factors and clinical out come after hematopoietic stem cell transplantation. Transplantation Proceedings 2010; 42: 3273-6.

6. Leather HL, Wingard JR. Infections following hematopoietic stem cell transplantation. Infect Dis Clin North Am. Jun 2001; 15 (2): 483-520.

7. Rapaport D, Ehgelhard D, Tagger G, et al. Antiviral prophylaxis may prevent human herpesvirus- 6 reactivation in bone marrow transplant recipients. Transplant Infectious Diseases 2002; 4: 10-16.

8. Rupesh Chawla H, Dele Davies. Infections After Bone Marrow Transplantation, aprile 2009. Online su: emedicine.medscape.com.

9. Sable CA, Donowitz GR. Infections in bone marrow transplant recipients. Clin Infect Dis 1994; 18 (3): 273-81.

10. Van Burik J, Weisdorf D. Infections in recipients of blood and marrow transplantation. In: Mandell GL, Bennett JE, Dolin R, eds. Principles and Practice of Infectious Diseases. $5^{\text {th }}$ ed. Philadelphia, Pa: Churchill Livingstone; 2000; 3136-47.

11. Yahav D, Gafter-Gvili A, Muchtar E, et al. Antiviral prophylaxis in haematological patients: systematic review and meta-analysis. Eur J Cancer 2009; 45: 3131-48. 